\title{
Nintedanib-mediated improvement in CT imaging in pulmonary fibrosis associated with systemic scleroderma
}

\begin{abstract}
Nintedanib is an antifibrotic drug that has an inhibitory effect on growth factor tyrosine kinases. In patients with idiopathic pulmonary fibrosis and systemic scleroderma-associated interstitial pneumonia (SSc-IP), nintedanib has been effective in suppressing the decline in forced vital capacity over time and the onset of acute exacerbation of interstitial pneumonia. Here, we report a SSc-IP patient who showed an improvement on CT images following nintedanib treatment. To our knowledge, this is the first report of such a case. Although SSc-IP patients are very rare, additional clinical experience and understanding will be required to prove the therapeutic benefit of nintedanib in these cases in relation to improved chest images.
\end{abstract}

Key words: systemic scleroderma, pulmonary fibrosis, nintedanib, computed tomography

Adv Respir Med. 2021; 89: 528-531

\section{Introduction}

Systemic scleroderma-associated interstitial pneumonia (SSc-IP) is a collagen disease. It is characterized by organ fibrosis due to fibroblast proliferation and impaired blood circulation due to vascular endothelial cell proliferation in the skin and organs, such as the lung and kidney [1]. More than half of patients with SSc develop interstitial pneumonia (IP) due to inflammation and fibrosis in the lung interstitium [2, 3]. Acute or subacute progression of IP and pulmonary hypertension are serious pathological conditions that determine the prognosis of SSc patients [3].

The antifibrotic drug nintedanib has been shown to suppress the gradual decline in forced vital capacity (FVC) and the onset of acute exacerbation in idiopathic interstitial pneumonia (IPF) patients, and as such, has become one of the most reliable drugs for the treatment of IPF [4-6]. Similar clinical effects have recently been confirmed in patients with SSc-IP in a large-scale clinical trial [7]. While nintedanib-mediated suppression of progression and advance in prognosis have been broadly shown, an improvement on computed tomography (CT) imaging, however, has been reported in only a few IPF patients [8-10]. To the best of our knowledge, there have been no reports of a SSc-IP patient showing improvement on CT images. Here, we report on our treatment with nintedanib of a patient who exhibited improved CT imaging. Although this type of case is extremely rare, reporting it provides valuable suggestions for future treatment plans involving nintedanib.

\section{Material and methods}

A 73-year-old woman was referred to our hospital with ground-glass opacities in both lower lobes in a chest radiograph taken at mass screening. She had had slight dyspnea on exertion for several months. She was diagnosed as having SSc due to symmetrical thickening, tightening and induration of the skin of the fingers and the skin proximal to the metacarpophalangeal or metatarsophalangeal joints, reticular densities most pronounced in the basilar areas of the lungs on chest radiograph, and the presence of anti-centromere antibody. She had no smoking habit. The patient was pathologically diagnosed with primary biliary cholangitis 25 years ago.

Address for correspondence: Hiroaki Satoh, Division of Respiratory Medicine, Mito Medical Center, University of Tsukuba-Mito Kyodo General Hospital, Japan;

e-mail: hirosato@md.tsukuba.ac.jp

DOI: 10.5603/ARM.a2021.0072 | Received: 20.05.2021 | Copyright (C) 2021 PTChP | ISSN 2451-4934 | e-ISSN 2543-6031

This article is available in open access under Creative Common Attribution-Non-Commercial-No Derivatives 4.0 International (CC BY-NC-ND 4.0) license, allowing to download articles and share them with others as long as they credit the authors and the publisher, but without permission to change them in any way or use them commercially. 

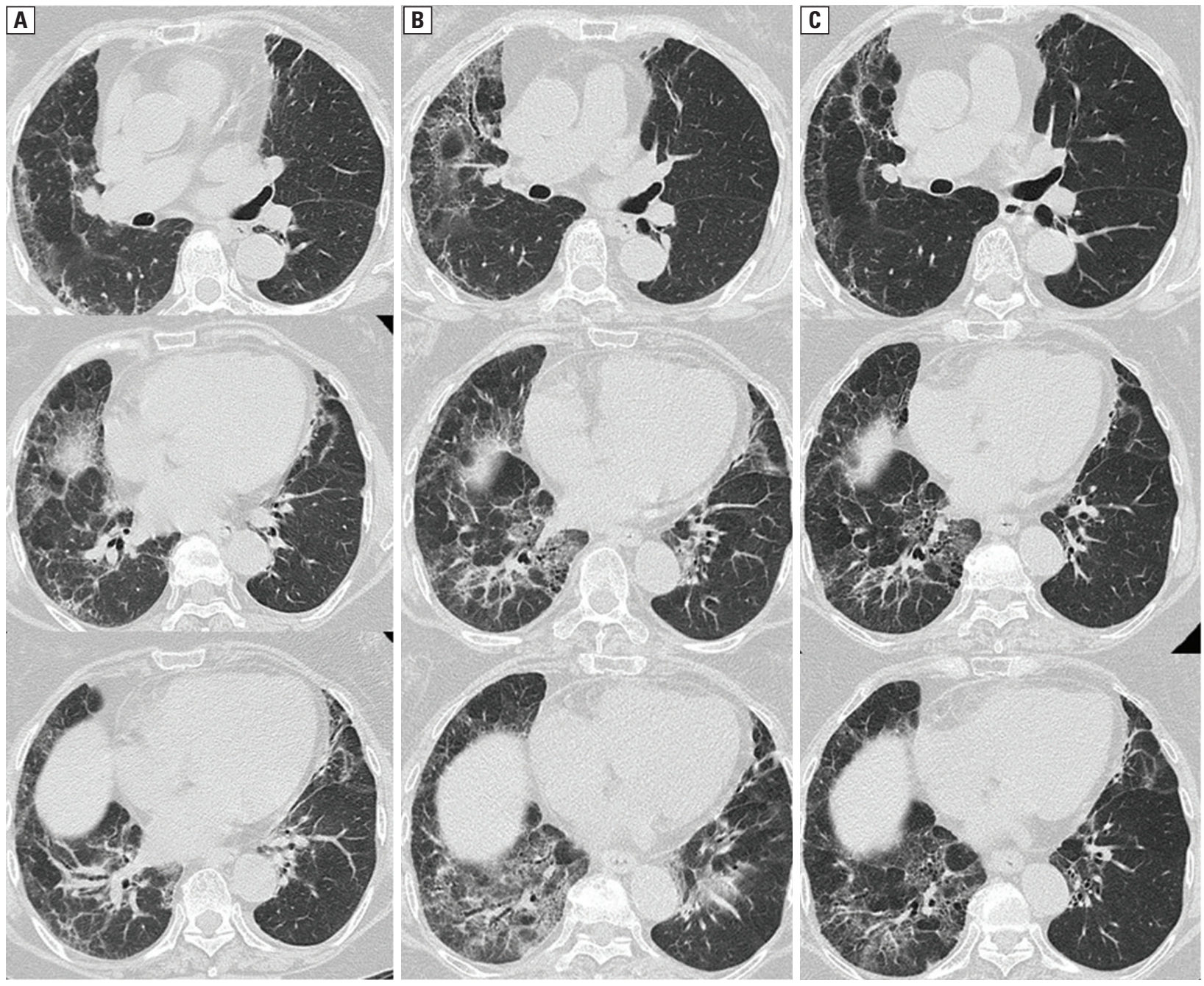

Figure 1. Chest computed tomography (CT) scan 10 months (A) and 26 months (B) after the initial visit to our hospital showed expansion of subpleural ground-glass opacities with thickening of vascular bundle in bilateral lower lobes of the lung. Chest CT scan taken 8 months after the initiation of nintedanib revealed an improvement of diffuse subpleural ground-glass opacities (C)

Computed tomography taken at the first visit showed traction bronchiectasis, bronchial bundle thickening, and ground-glass opacities in the lower lobes of both lungs. Blood examination at the first visit revealed thrombocytopenia, which was refined to idiopathic thrombocytopenia after more testing results.

Therapy with prednisolone and tacrolimus was started as a treatment for idiopathic thrombocytopenic purpura. The tacrolimus dose was $2 \mathrm{mg} /$ day, commencing two years before the initiation of nintedanib. Prednisolone was reduced to $5 \mathrm{mg} /$ day four months before the initiation of nintedanib, with this dose remaining constant ever since. Chest CT images taken 10 months and 26 months after the initial visit to our hospital showed the range of ground-glass opacities expanded and exacerbation was confirmed (Figure $1 \mathrm{~A}$ and $1 \mathrm{~B})$.
At that time, nintedanib was reimbursed through health insurance in our country. Patients were thoroughly informed on the benefits and adverse effects of nintedanib before starting nintedanib treatment. In particular, the dose of nintedanib was explained in relation to the results of the SENSCIS study. Treatment started at $2 \times 100 \mathrm{mg}$ of nintedanib daily. One month after the administration of nintedanib, the patient noticed an improvement in her dry cough and dyspnea, and due to this amelioration in clinical symptoms and imaging, desired to continue on the same nintedanib dose. As a result, there was no dose increase. There were no side effects such as loss of appetite or nausea. After commencing nintedanib treatment, the patient was asked several times about the presence or absence of diarrhea, but there were no side effects of diarrhea, probably because the patient was taking codeine 
phosphate as an antitussive. Blood sampling examinations including liver function were performed regularly, but no abnormal values due to nintedanib were found. Chest CT was performed eight months after the start of nintedanib, and a regression of ground-glass opacities on chest CT scan was confirmed (Figure 1C). An improvement on chest CT scan was accompanied by a decrease in dry cough and dyspnea on exertion. One year after the start of nintedanib, treatment is ongoing.

\section{Discussion}

In many pulmonary fibrosis cases, including IPF and SSc-IP, fibrosis progresses due to an abnormal repair reaction to damage to the alveolar epithelium. During this abnormal fibrosis, alveolar epithelial cells differentiate into fibroblasts and myofibroblasts [11]. Lung fibroblast proliferation and migration is stimulated by fibroblast growth factor (FGF) and vascular endothelial growth factor (VEGF), and fibroblast transformation is induced by transforming growth factor- $\beta 2$ [11]. Nintedanib inhibits the action of growth factors on tyrosine kinases, mainly FGF, VEGF, and platelet derived growth factor (PDGF). In a phase III trial in IPF patients, nintedanib was effective in suppressing the decline in FVC over time and the onset of acute exacerbation of IP [4-6]. In SSc-IP patients, a large-scale clinical trial has recently been conducted [7], and the clinical effects observed in these subjects are similar to that observed in IPF patients [12]. There are only a few reported cases that show an improvement on CT images with nintedanib therapy, and all are patients with IPF [8-10].

A recent sub-study analysis of the SENSCIS trial examined the effect of nintedanib on HRCT markers of fibrosis [13]. It found only nine out of 52 SSc-ILD patients treated with nintedanib had a notable improvement in fibrotic changes on HRCT analysis, and none were classified as 'much better', which was defined by the authors as 'a moderate decrease in honeycombing and/or reticulation and/or fibrotic ground-glass opacity; a decrease was more than 10\%'. According to this sub-study analysis, an improvement on CT was extremely rare.

To the best of our knowledge, no similar cases of HRCT improvement in SSc-ILD after nintedanib therapy have been published to date. Therefore, this is the first SSc-IP case who has showed an amelioration in CT images following nintedanib treatment, defined by a regression of ground-glass opacities and septal thickening on the chest CT scan. Our patient also showed an improvement in her dry cough as well as dyspnea on effort.

It is important to consider the possibility that mechanisms other than nintedanib might have contributed to the patient's improved CT images. Firstly, there is the possibility of spontaneous changes in SSc cases [14]. Spontaneous changes in skin lesions have been reported in SSc patients, but there have been no reports of improvement in opacities on chest CT images. Secondly, there is the potential effect of drugs other than nintedanib. The patient was also receiving corticosteroids and tacrolimus, which both commenced three years before the initiation of nintedanib. The dose of tacrolimus had been constant for two years before the initiation of nintedanib. The dose of prednisolone was reduced four months before the introduction of nintedanib, and had not changed since. Chest CT scans were taken several times during the administration of these drugs, which showed a gradual exacerbation of ground-glass opacities over time, but no improvement. Therefore, the possibility of these drugs contributing to the improvement on CT images is low, and it was instead considered to be due to the effect of nintedanib.

Nintedanib has been reported to have gastrointestinal adverse effects (AEs) such as appetite loss, nausea and liver dysfunction [4-6]. In our patient, frequent examination did not find any severe AEs to prevent continuing treatment. In addition to CT images, therapeutic effects in SScIP patients can be observed in respiratory function and pathological evaluations. On exertion, there was no decrease in oxygen saturation, and an improvement in subjective symptoms such as dry cough and dyspnea was observed. However, as the patient did not wish to be examined, there were no assessment on pulmonary function and histopathological evaluation.

This case report has some limitations. One of them is the lack of pathological examination. The pattern shown on CT scans was probably nonspecific interstitial pneumonia, and this pattern might be reversible, if cellular component was present. Evaluation of tissue specimens obtained by transbronchial lung biopsy (TBLB) would be useful. If performing TBLB was difficult, analysis of bronchoalveolar lavage fluid would be helpful to understand why these changes were reversible. The other serious disadvantage was the lack of lung function data before and after treatment. Results of pulmonary function tests, including spirometry and diffusing capacity for carbon monoxide, and six-minute walk test before and 
after eight months of therapy with nintedanib, would be important to confirm a functional improvement corresponding to a CT amelioration of fibrotic changes. However, due to patient's refusal to perform any of these tests, as well as the circumstances associated with the outbreak of coronavirus disease in 2019 (COVID-19), they were not performed.

\section{Conclusions}

In conclusion, our observation suggests that nintedanib contributes to reducing regression of ground-glass opacities in SSc-IP patients. This indicates these rare patients could be expected to exhibit improved CT images with nintedanib therapy. It remains to be elucidated what characteristics of SSc-IP patients are related to improvement of the CT image, and the precise mechanism of action of nintedanib noting its inhibitory action on growth factors such as FGF, VEGF, and PDGF. Although careful consideration of side effects is required, if nintedanib can improve CT images in addition to the previously confirmed effects, it would be a desirable treatment option for SScIP patients. Additional clinical experience and knowledge will be required to prove our observation of the therapeutic effect of nintedanib on improving chest images.

\section{Statement of ethics}

This study was approved by the institutional ethics committee of each Hospital (Project approval number: NO16-66). Written comprehensive informed consent at the time of admission for obtaining pathological specimens was obtained from the patient.

\section{Conflict of interest}

None declared.

\section{References:}

1. van den Hoogen F, Khanna D, Fransen J, et al. 2013 classification criteria for systemic sclerosis: an American college of rheumatology/European league against rheumatism collabora- tive initiative. Arthritis Rheum. 2013; 65(11): 2737-2747, doi: 10.1002/art.38098, indexed in Pubmed: 24122180.

2. Walker UA, Tyndall A, Czirják L, et al. Clinical risk assessment of organ manifestations in systemic sclerosis: a report from the EULAR Scleroderma Trials And Research group database. Ann Rheum Dis. 2007; 66(6): 754-763, doi: 10.1136/ ard.2006.062901, indexed in Pubmed: 17234652.

3. Elhai M, Meune C, Boubaya M, et al. EUSTAR group. Trends in mortality in patients with systemic sclerosis over 40 years: a systematic review and meta-analysis of cohort studies. Rheumatology (Oxford). 2012; 51(6): 1017-1026, doi: 10.1093/rheumatology/ker269, indexed in Pubmed: 21900368.

4. Richeldi L, du Bois RM, Raghu G, et al. INPULSIS Trial Investigators. Efficacy and safety of nintedanib in idiopathic pulmonary fibrosis. N Engl J Med. 2014; 370(22): 2071-2082, doi: 10.1056/NEJMoa1402584, indexed in Pubmed: 24836310.

5. Collard HR, Richeldi L, Kim DS, et al. INPULSIS Trial Investigators. Efficacy and safety of nintedanib in idiopathic pulmonary fibrosis. N Engl J Med. 2014; 370(22): 2071-2082, doi: 10.1056/NEJMoa1402584, indexed in Pubmed: 24836310.

6. Crestani B, Huggins JT, Kaye M, et al. Long-term safety and tolerability of nintedanib in patients with idiopathic pulmonary fibrosis: results from the open-label extension study, INPULSIS-ON. Lancet Respir Med. 2019; 7(1): 60-68, doi: 10.1016/ S2213-2600(18)30339-4, indexed in Pubmed: 30224318.

7. Maher TM, Mayes MD, Kreuter M, et al. SENSCIS Trial Investigators. Effect of nintedanib on lung function in patients with systemic sclerosis-associated interstitial lung disease: further analyses of a randomized, double-blind, placebo-controlled trial. Arthritis Rheumatol. 2021; 73(4): 671-676, doi: $10.1002 /$ art.41576, indexed in Pubmed: 33142016.

8. Ito Y, Tazaki G, Kondo Y, et al. Therapeutic effect of nintedanib on acute exacerbation of interstitial lung diseases. Respir Med Case Rep. 2019; 26: 317-320, doi: 10.1016/i.rmcr.2019.02.021, indexed in Pubmed: 30931251.

9. Nakano A, Ohkubo H, Fukumitsu K, et al. Remarkable improvement in a patient with idiopathic pulmonary fibrosis after treatment with nintedanib. Intern Med. 2019; 58(8): 1141-1144, doi: 10.2169/internalmedicine.1890-18, indexed in Pubmed: 30568147.

10. Tomioka H, Takata $H$. Treatment with nintedanib for acute exacerbation of idiopathic pulmonary fibrosis. Respirol Case Rep. 2017; 5(2): e00215, doi: 10.1002/rcr2.215, indexed in Pubmed: 28096998.

11. Hilberg F, Roth GJ, Krssak M, et al. BIBF 1120: triple angiokinase inhibitor with sustained receptor blockade and good antitumor efficacy. Cancer Res. 2008; 68(12): 4774-4782, doi: 10.1158/00085472.CAN-07-6307, indexed in Pubmed: 18559524.

12. Fischer A, Distler J. Progressive fibrosing interstitial lung disease associated with systemic autoimmune diseases. Clin Rheumatol. 2019; 38(10): 2673-2681, doi: 10.1007/s10067019-04720-0, indexed in Pubmed: 31423560.

13. Hachula E, Hamblin M, Ogura T et al. Changes in imaging markers in patients with systemic sclerosis-associated interstitial lung disease (SSc-ILD) treated with nintedanib: Substudy of the SENSCIS Trial. Abstract Number: 1382, ACR Convergence 2020. https://acrabstracts.org/abstract/changes-in-imaging-markers-in-patients-with-systemic-sclerosis-associated-interstitial-lung-disease-ssc-ild-treated-with-nintedanib-sub-study-of-the-senscis-trial/.

14. Schneider PD, Wise RA, Hochberg MC, et al. Serial pulmonary function in systemic sclerosis. Am J Med. 1982; 73(3): 385394, doi: 10.1016/0002-9343(82)90732-x, indexed in Pubmed: 7124766. 